\title{
Recent Progress in Dual-Beam Microscopy 3D Imaging
}

\author{
Z. G. Li
}

DuPont Central Research \& Development, P. O. Box 80228, Wilmington, DE 19880-0228

It is apparent now that the new dual-beam (DB) microscopy has become an important microstructure characterization technique and possesses many unique capabilities which conventional TEM and SEM lack [1-2]. DB microscopy has been used to characterize many very different materials, including inorganic, organic/inorganic composite, and biological materials. The DB microscopy technique combines the power of ultra-high resolution field emission scanning electron microscopy and precise focused ion beam together on a single platform. While the electron beam images a selected area, the focused ion beam acts as a micro-surgical tool, allowing us to view its internal structure. This unique capability overcomes the limits of top-surface imaging in SEM and DB microscopy 3D images can be reconstructed.

Figure 1 shows the principle of DB microscopy 3D imaging in more detail. The focused ion beam first cuts a plane perpendicular to the surface of a sample. Then the electron beam is used to image the microstructure of the focused ion beam cut surface. By automatically repeating the cutting and imaging process, a stack of 2D digital images are collected. The technique is referred as the "slicing and viewing" approach, very different from other 3D imaging techniques. The 3D images can be reconstructed by a software available in the market. The contrast between the different components in a material is quite good due to the excellent design of imaging and detector systems. The resolution of 3D imaging in z-direction depends directly on the size of the focused ion beam (cutting thickness). Currently, $10 \mathrm{~nm}$ or better resolution can be routinely achieved. Because both ion-beam and electron beams are scanned fast enough, the total time to collect 3D images is very reasonable for real-world applications. As an example, figure 2 shows three of DB microscopy 3D images of organic/inorganic composite materials.

Applications of DB microscopy 3D imaging in many fields have been reported. Lee et al. generated 160 serial sectioned DB microscopy 3D images of Ni based superalloys with $\mathrm{x}-\mathrm{y}$ dimensions of about $15 \times 13$ microns [3]. Their 3D image was reconstructed with the IMOD program developed at the University of Colorado-Boulder. DB microscopy also plays a key role in sample preparation for high resolution 3D STEM tomography [4]. We would like to mention that the significant progress has been made in the 3D chemical analysis of the materials, reported by Kotula et al. [5]. By comparing it with other 3D imaging techniques, we will review the advantages and limitation of DB microscopy 3D imaging technique.

References

1. L. A. Giannuzzi and F. A. Stevie, Editors, Introduction to Focused Ion Beams. Instrumentation, Theory, Techniques and Practice, Springer, New York (2005).

2. Y. Xu and C. Schwappach, in Industrial Applications of Electron Microscopy, ed. by Z. G. Li, Marcel Dekker, New York (2003) 153.

3. E. Lee et al., Microsc. Microanal. 10 (supp12) (2004) 1128.

4. S. M. Schwarz and L. A. Ginnuzzi, Microsc. Microanal. 10 (suppl2) (2004) 142. 
5. P. G. Kutula et al., Microsc. Microanal. 10 (suppl2) (2004) 1132.

1a)

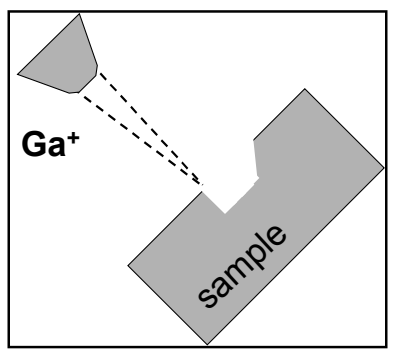

1b)

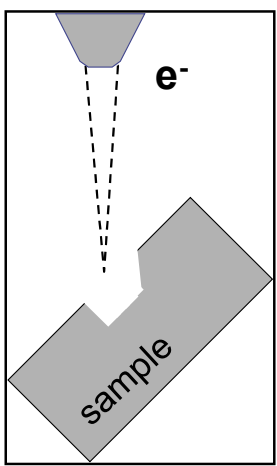

1c)

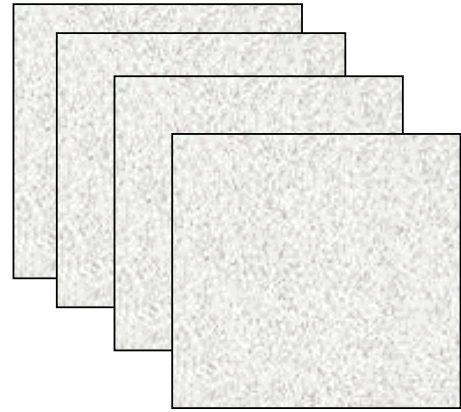

Figure 1. Schematic representation of DB microscopy 3D imaging. a) focused ion beam cut a plane perpendicular to the surface; then b) electron beam is used to image the cut surface; c) by repeating the cutting and imaging process, a stack of $2 \mathrm{D}$ images are collected.
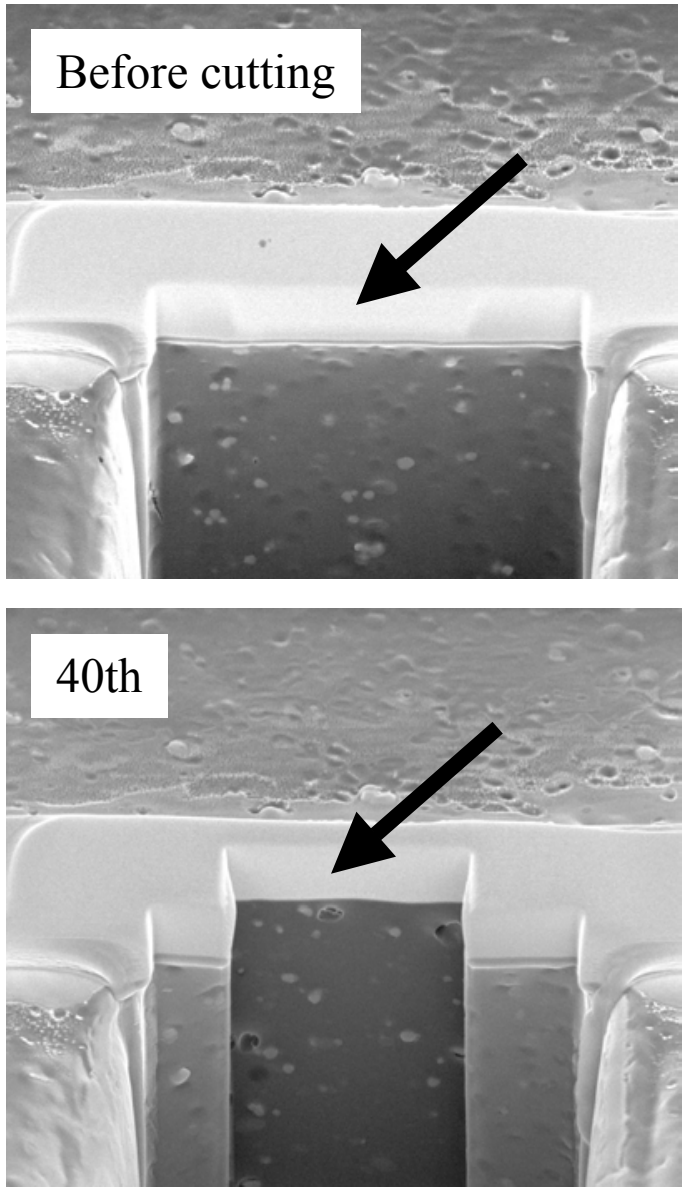

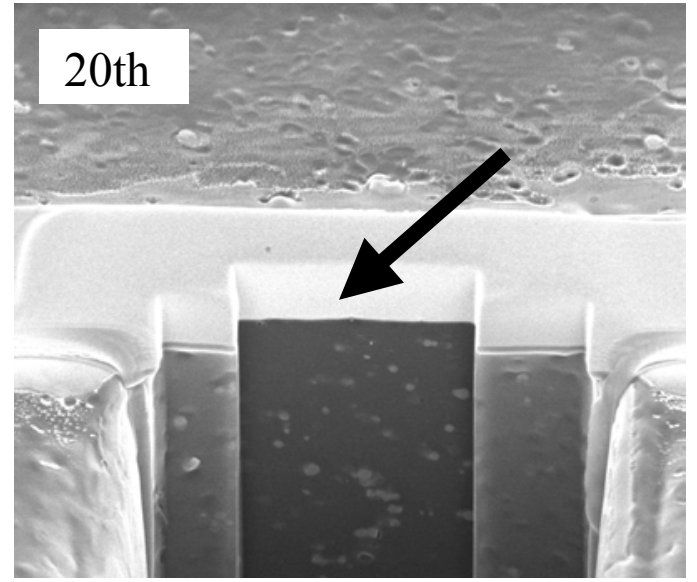

Fig. 2. Three of DB microscopy images of organic/inorganic composite material. 3D images of the interested areas indicated by arrows can be reconstructed by a software. 